\title{
ANÁLISE DE CAUSA RAIZ EM INTERNAÇÕES POR DROGAS DE ABUSO COMO EVENTOS SENTINELAS
}

\section{ROOT CAUSE ANALYSIS IN HOSPITALIZATIONS DUE TO DRUGS AS SENTINEL SURVEILLANCE}

\section{ANÁLISIS DE CAUSA RAÍZ EM INTERNACIONES POR DROGAS DE ABUSO COMO EVENTOS CENTINELAS}

Cleiton José Santana ${ }^{1}$, Rosangela Cristophoro², Mirella Machado Ortiz ${ }^{3}$, Denise Raquel dos Santos ${ }^{4}$, Lúcia Margarete dos Reis ${ }^{5}$, Magda Lúcia Félix de Oliveira ${ }^{6}$

\section{RESUMO}

Objetivo: Analisar os fatores de iniciação e continuidade do uso de drogas, a partir do evento sentinela, internação hospitalar com diagnóstico de trauma associado à intoxicação por drogas de abuso. Métodos: Estudo exploratório e retrospectivo, com referencial de vigilância epidemiológica de 30 eventos sentinelas, a partir de revisão de documentos hospitalares e entrevista com familiar dos usuários de drogas. A matriz para avaliação foi a análise da causa raiz, estabelecida pela investigação e pelar e construção da trajetória do uso de drogas. Resultados: Houve predomínio de sexo masculino, baixa escolaridade e desemprego, com média de 40,1 anos. A droga mais utilizada foi o álcool, isolada ou associada a drogas ilícitas. Identificaram-se pontos críticos de vulnerabilidade social, permitindo uma discussão sobre o desempenho das políticas públicas. Conclusão: A maioria dos fatores subjacentes se relacionava à ausência ou à precariedade de políticas públicas, com pontos críticos na intersetorialidade.

Descritores: Vigilância de evento sentinela; Drogas ilícitas; Saúde mental; Vulnerabilidade social; Política pública.

\section{ABSTRACT}

Objective: to analyze the initiation and continuity factors for drug use from the hospitalization sentinel surveillance diagnosed with trauma associated with drug intoxication. Methods: this is an exploratory and retrospective study with the epidemiological surveillance framework of 30 sentinel events based on hospital documents review and interviews with family members of drug users. The matrix for the evaluation was the root cause analysis, established by the investigation and reconstruction of the drug use trajectory. Results: we found predominance of male gender, low education and unemployment, with average 40.1 years. The most consumed drug was alcohol, alone or associated with illicit drugs. Critical points of social vulnerability were identified, admitting the discussion of public policies performance. Conclusions: most of the underlying factors were related to the absence or precariousness of public policies, with critical points in intersectoriality.

Descriptors: Sentinel surveillance; Illicit drugs; Mental health; Social vulnerability; Public policy.

\section{RESUMEN}

Objetivo: analizar los factores de iniciación y continuidad para el consumo de drogas del evento centinela de hospitalización, diagnosticado con trauma asociado con la intoxicación por abuso de drogas. Métodos: estudio exploratorio y retrospectivo con el marco de vigilancia epidemiológica de 30 eventos centinela, basado en la revisión de documentos hospitalarios y entrevistas con familiares de usuarios de drogas. La matriz para la evaluación fue el análisis de la causa raíz, establecido por la investigación y reconstrucción de la trayectoria del consumo de drogas. Resultados: predominó el sexo masculino, baja educación y desempleo, con un promedio de 40,1 años. La droga más utilizada fue el alcohol, solo o asociado con drogas ilícitas. Se identificaron puntos críticos de vulnerabilidad social, lo que permite una discusión sobre el desempeño de las políticas públicas. Conclusión: la mayoría de los factores subyacentes estaban relacionados con la ausencia o precariedad de las políticas públicas, con puntos críticos en la intersectorialidad.

Descriptores: Vigilancia de guardia; Drogas ilícitas; Salud mental; Vulnerabilidad social; Política pública.

${ }^{1}$ Enfermeiro, Doutorando em Enfermagem pelo Programa de Pós Graduação em Enfermagem da Universidade Estadual de Maringá (PSE/UEM). Docente Faculdade Pitágoras Campus Londrina. ${ }^{2}$ Enfermeira, Doutora em Enfermagem pelo Programa de PSE/UEM. Docente do Departamento de Enfermagem da UEM. ${ }^{3}$ Enfermeira, Doutoranda em Enfermagem pelo Programa de PSE/UEM. ${ }^{4}$ Graduanda em Enfermagem pela UEM. ${ }^{5}$ Enfermeira, Doutora em Enfermagem, Coordenadora do CAPS de Sarandi. ${ }^{6}$ Enfermeira. Doutora em Saúde Coletiva. Professora do Departamento de Enfermagem da Universidade Estadual de Maringá.

\section{Como citar este artigo:}

Santana CJ, Cristophoro R, Ortiz MM, et al. Analise de Causa Raiz em Internações por Drogas de Abuso como Eventos Sentinela. Revista de Enfermagem do Centro Oeste Mineiro 2020;10:e3542. [Access DOI: http//doi.org/10.19175/recom.v10i0.3542. \}. Available in: 


\section{INTRODUÇÃO}

A saúde pública volta-se, cada vez mais, para a vigilância e o monitoramento de agravos e doenças como ferramentas de orientação no planejamento e na avaliação de programas e políticas de saúde ${ }^{(1)}$. No Brasil, entre os desafios da vigilância em saúde, estão o desenvolvimento de novos sistemas de informação e as novas abordagens para coletar dados de vigilância, em tempo real, principalmente para alcançar populações vulneráveis ou que não podem ser facilmente identificadas, por meio de variáveis demográficas padronizadas. São necessários novos sistemas, para o rastreamento de medicamentos prescritos, além da expansão dos sistemas existentes, para a vigilância de saúde mental e do uso de drogas de abuso seja incorporado ${ }^{(2)}$.

As drogas, como substâncias introduzidas no organismo, que causam alteração em seu funcionamento, modificando funções vitais e comportamentos, começaram a ser consideradas um problema social, a partir das grandes mudanças socioeconômicas e dos prejuízos advindos de seu uso abusivo. A utilização de drogas pode ocorrer como meio de obtenção de prazer; para aliviar a ansiedade, o estresse e os medos; e até para abrandar dores físicas ${ }^{(3-4)}$.

Usuários de drogas, muitas vezes, estão em vulnerabilidade relacionada a fatores individuais, socioeconômicos e culturais, exigindo um olhar aprofundado e ciente de sua complexidade. Pensar na atenção às necessidades das populações vulneráveis é um desafio para os profissionais de saúde, na busca de oferecer não apenas cuidados técnicos, mas também assistência que configure a real possibilidade de promover transformações nas políticas públicas existentes ${ }^{(5-6)}$.

Apesar de se reconhecer que o uso das drogas é um problema social e de saúde pública emergente, o emprego da epidemiologia como instrumento de detecção e avaliação do consumo dessas substâncias é recente. Conhecer o padrão do consumo de drogas de uma dada sociedade é imprescindível para a implantação de programas de prevenção ao consumo, pois informações atualizadas fornecem parâmetros para políticas públicas voltadas à prevenção e ao tratamento ${ }^{(7-8)}$.

No entanto, não existe um processo de vigilância epidemiológica efetivo para mensuração do efeito das drogas de abuso na saúde da população. Dados de unidades sentinelas ou áreas sentinelas ou de inquéritos de base populacional são utilizados para pautar as intervenções de prevenção e cuidado, mas têm se mostrado insuficientes para reorientar as ações em nível local, apoiar programas de intervenção e servir como contraponto à avaliação de outros agravos associados e mais suscetíveis a intervenções ${ }^{(9-10)}$.

A vigilância epidemiológica é a contínua e sistemática coleta, análise e interpretação de dados sobre eventos que afetam a população, desenvolvida a partir de sistemas locais de saúde, com o objetivo de agilizar o processo de identificação e controle de eventos adversos à saúde ou de fatores de risco(11). Em sistemas de vigilância epidemiológica, a coleta de dados pode ser realizada por métodos passivos e espontâneos de notificação e por métodos ativos, que requerem contato a intervalos regulares entre a saúde e as fontes de informação, permitindo um melhor conhecimento do comportamento dos agravos à saúde na comunidade, em seus aspectos tanto quantitativos quanto qualitativos ${ }^{(12)}$.

Uma das metodologias ativas para a vigilância em epidemiologia é o evento sentinela, que se aplica à detecção de doença prevenível, à incapacidade ou à morte inesperada, cuja ocorrência serve como sinal de alerta de que a qualidade da assistência deve ser questionada. Essas condições poderiam ser melhoradas por tecnologias e ações de saúde eficazes, e constituem-se como clearcuttings, ou seja, índices da qualidade da assistência à saúde de uso imediato $^{(13-14)}$. A formação de sistemas de vigilância de eventos sentinelas tem como objetivo monitorar indicadores-chaves na população geral ou em grupos especiais. A seleção de um fato negativo, com resultado adverso sério, sinaliza a necessidade imediata de resposta e gera dois desafios: compreender como e por que o evento ocorreu, e prevenir a ocorrência do mesmo evento ou de evento similar ${ }^{(13-15)}$.

Em 1998, o Ministério da Saúde já estimulava e apoiava a utilização de eventos sentinelas, visando a obter informações capazes de atender ao principal objetivo da vigilância epidemiológica, que é o pronto desencadeamento de ações preventivas ${ }^{(16)}$. Desde 2007, um grupo de pesquisadores de enfermagem. do Paraná, tem considerado, de forma experimental, eventos sentinelas como uma ferramenta para a vigilância epidemiológica das intoxicações e dos efeitos das drogas de abuso na saúde humana, com definição de caso/evento, operacionalização da investigação epidemiológica e testes para a avaliação da potencialidade do evento, com vistas 
à institucionalização da vigilância em um sistema local de saúde ${ }^{(17-21)}$.

Embora de forma desigual nas diferentes regiões, o monitoramento da relação entre drogas de abuso, violência e trauma é realizado em nosso país, a partir de informações de alguns Centros de Informação e Assistência Toxicológica (CIAT), considerados unidades sentinelas para 0 monitoramento das intoxicações. No entanto, o procedimento de vigilância epidemiológica, por meio de fontes ou eventos sentinelas, visando a estabelecer a causa básica do evento (raiz causal) ou riscos evitáveis e a propor futuras medidas preventivas, ainda é pouco utilizado no Brasil ${ }^{(12,22)}$.

Geralmente, usuários de drogas de abuso acessam os serviços de saúde, principalmente a Rede de Atenção às Urgências, quando apresentam complicações relacionadas ao consumo compulsivo, com comprometimento clínico, decorrente do uso crônico ou em situações de violência e trauma. Para a vigilância epidemiológica do efeitos associados às drogas de abuso na saúde, devem ser utilizados como evento sentinela, todos os casos de pessoas internadas em unidades de atenção às urgências com diagnóstico de trauma físico associado à intoxicação por drogas de abuso.

O presente estudo teve início, a partir da premissa de que a intercorrência internação por trauma em prontos-socorros foi entendida como evitável, configurando indicador de gravidade das condições de vida dos usuários de drogas e de suas famílias, podendo ser relacionada com possíveis falhas do cuidado à saúde ou insuficiências dos serviços básicos de saúde ${ }^{(13-14,20)}$.

A questão norteadora da presente investigação foi a seguinte: Qual é, afinal, o evento sentinela desencadeante por detrás do uso e o abuso de drogas em indivíduos internados por trauma associado à intoxicação por drogas de abuso?

O objetivo foi de analisar os fatores para a iniciação e a continuidade do uso de drogas, a partir do evento sentinela internação hospitalar com diagnóstico de trauma associado à intoxicação por drogas de abuso.

\section{MÉTODO}

Estudo descritivo e retrospectivo, tomandose como referencial a investigação epidemiológica de eventos sentinelas ${ }^{(13-14)}$ e, como método de avaliação dos casos, a análise de causa raiz (RCA, do inglês root cause analysis) ${ }^{(23)}$.

A investigação epidemiológica de eventos sentinelas pressupõe identificar eventos graves que poderiam ser evitados por políticas públicas eficazes ${ }^{(13-14)}$. A RCA é o processo sistemático, realizado após a ocorrência de eventos sentinelas, em que os fatores que contribuíram para o evento são identificados pela reconstrução da sequência de eventos e pelo constante questionamento do porquê, buscando reduzir ou eliminar o risco de recorrências ${ }^{(23-24)}$. A análise se aprofunda, perguntando "o que" e "por que" até que todos os aspectos dos processos sejam analisados, e sejam considerados os fatores que contribuíram para o evento $^{(22,24)}$. A matriz para avaliação dos casos seguiu o modelo RCA adaptado ${ }^{(22,24)}$.

O estudo foi realizado, no município de Maringá (PR) e se deu pela verificação de casos originados da unidade de atenção às urgências de um hospital ensino e notificados como intoxicação alcoólica a um CIAT, denominado Centro de Controle de Intoxicações, no período de abril a setembro de 2014.

A amostra foi intencional, formada por pacientes que se enquadravam no escopo do evento sentinela proposto, ou seja, com quadro clínico compatível ou testes laboratoriais confirmativos de intoxicação por drogas de abuso associada à trauma físico; internados em um hospital ensino; acessados a partir da notificação a um CIAT, independentes de sexo e idade, com vínculo familiar e moradia definitiva, residentes no município de Maringá (pela dimensão locorregional da sistemática de vigilância epidemiológica). Os casos foram denominados eventos sentinelas, e um familiar foi considerado informante-chave para a investigação epidemiológica.

No período em estudo, foram encontrados 171 pacientes com registro de efeitos do uso de drogas de abuso. Destes, cem tinham diagnóstico médico de trauma associado ao uso de drogas e foram considerados eventos-sentinelas, sendo 50 residentes em Maringá. Considerando os critérios de inclusão e exclusão, as perdas e as recusas, foram elegíveis para o estudo 30 eventos sentinelas (Figura 1). 
Figura 1 - Fluxograma da pesquisa. Maringá (PR), Brasil, abril a setembro, 2014.

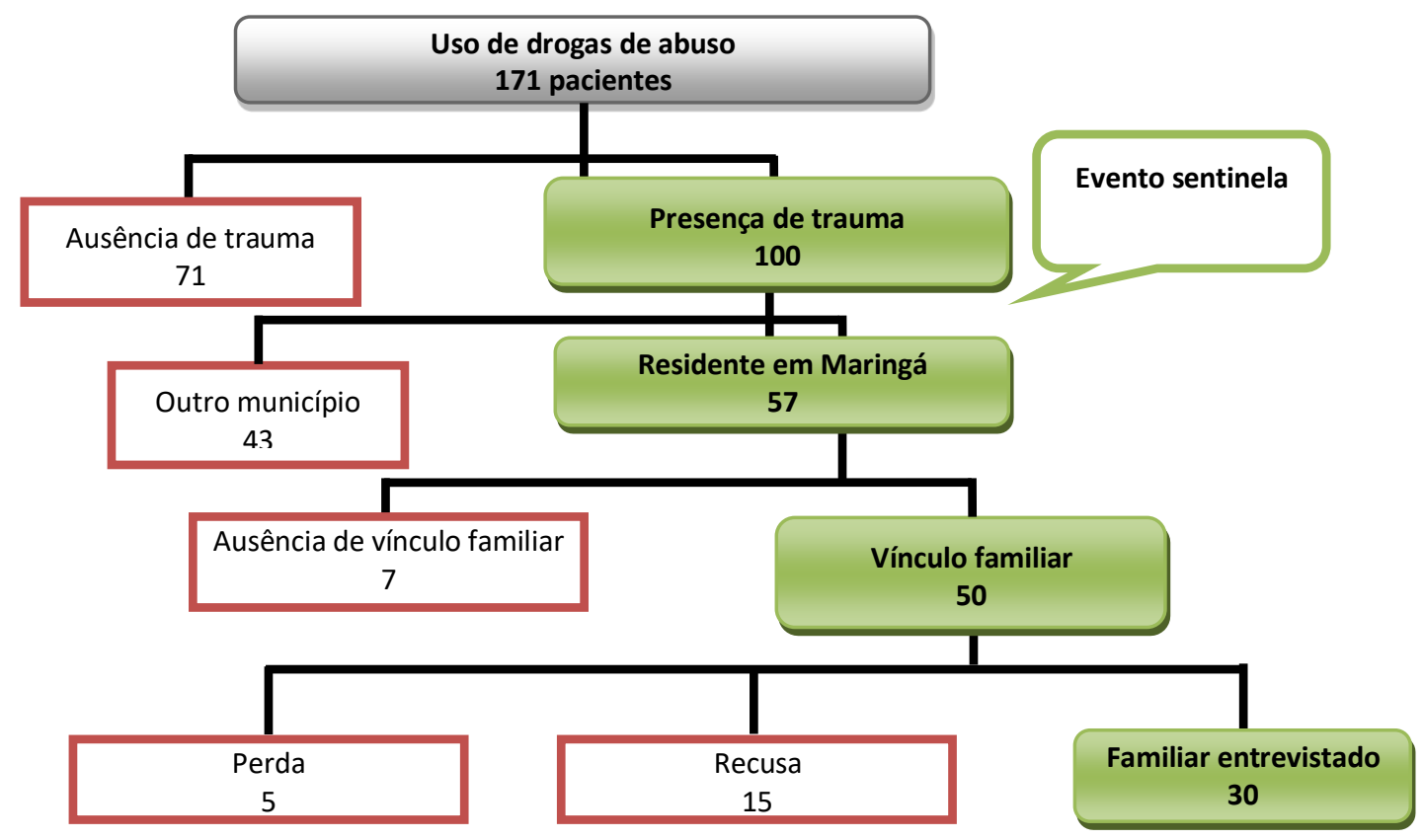

Documentos hospitalares e contato telefônico subsidiaram a confirmação dos critérios de inclusão. Os familiares foram convidados a participar da pesquisa por contato telefônico. Os casos em que se constatou ausência de vínculo familiar foram excluídos.

Foram utilizados a Ficha Epidemiológica de Ocorrência Toxicológica de Intoxicação Alcoólica e/ou outras Drogas de Abuso (ficha OT/IA), padronizada nacionalmente e arquivada no CIAT, e o prontuário hospitalar do paciente, para a confirmação diagnóstica e avaliação da internação hospitalar e do manejo clínico.

$O$ instrumento de coleta de dados foi o roteiro de investigação de eventos sentinelas, semiestruturado, composto por quatro blocos temáticos, que abordaram informações socioeconômicas e demográficas do usuário de drogas; evento sentinela e avaliação clínica; investigação domiciliar e familiar; e avaliação e conclusão do caso.

A coleta de dados seguiu a metodologia de investigação de eventos sentinelas, ampliada pela avaliação clínica dos casos. Os dados primários foram coletados em um único encontro com cada familiar, por meio de entrevistas domiciliares individuais com duração aproximada de 50 minutos.

Para a análise documental, foram listados, para estudo, a caracterização do evento sentinela, a história da intoxicação, o atendimento préhospitalar (local, tipo de ocorrência e droga de abuso), os dados da internação hospitalar e a classificação clínica do caso. Na entrevista com o familiar, foram abordados os seguintes pontos: condições sociodemográficas da família e os dados do usuário de drogas informados por familiar; comportamento do indivíduo no ambiente familiar, no trabalho e na vida social; data do início e motivação para o uso de drogas; tempo entre início do uso das drogas e descoberta da família; tempo de uso de drogas; história de tratamento para dependência; ciclo abstinência/recaída; e acesso às políticas públicas. Ainda na entrevista domiciliar com o familiar, seguindo a técnica recomendada para estudos de eventos sentinelas ${ }^{(13-14)}$, foi realizada a reconstrução da trajetória individual de cada caso, com dados da ocorrência toxicológica, da contextualização do atendimento e da internação hospitalar, da história aditiva familiar e do acesso das famílias às políticas psicossociais.

Por meio da análise sistemática da história de cada caso, após a busca dos registros nos prontuários hospitalares e nas fichas OT/IA, e a entrevista com o familiar, foram investigados os possíveis fatores que influenciaram o uso de droga e, consequentemente, a internação hospitalar com diagnóstico de trauma. Para resgatar essas informações, elaboraram- se, então, três perguntas: Por que o indivíduo iniciou o uso de drogas de abuso? Por que ele continuou o uso de drogas de abuso? Onde aconteceram fracassos?

Após a avaliação de cada caso, foi preenchido, no roteiro de investigação de eventos sentinelas, o item conclusão do caso, com a 
análise do motivo básico para o uso de drogas de abuso e fatores de risco/vulnerabilidade, como os antecedentes e fatores de risco no domicílio, no trabalho, na escola, no serviço de saúde; a existência de apoio de redes sociais e serviços públicos; e a possibilidade de o evento ser evitado e por quais medidas sociais, educativas, assistência à saúde e outras.

Os procedimentos éticos do presente estudo são representados pela aprovação do Comitê Permanente de Ética em Pesquisa com Seres Humanos (COPEP), da Universidade Estadual de Maringá (UEM), parecer favorável 458.185/2013, CAAE: 06218713.0.0000.0104, e pela assinatura do Termo de Consentimento Livre e Esclarecido dos participantes que aceitaram participar.

\section{RESULTADOS E DISCUSSÃO}

O perfil dos 30 eventos sentinelas apontou predomínio do sexo masculino, idades entre 13 e 65 anos e média de 40,1 anos, além de baixa escolaridade e desemprego. A droga mais utilizada foi o álcool, isolada ou associada a outras drogas e, metade deles, utilizavam-nas diariamente. A maioria dos eventos aconteceu em ambiente externo à residência $(73,4 \%)$, sendo, principalmente, acidentes de trânsito, quedas e agressões físicas. (Tabela 1)

Tabela 1 - Variáveis sociodemográficas e econômicas dos eventos sentinelas. Maringá (PR), Brasil, abril a setembro, 2014.

\begin{tabular}{lc}
\hline \hline Variáveis & Resultados \\
\hline Sexo masculino & 96,7 \\
Raça/cor branca (declarada) & 66,7 \\
Católico (não praticante) & 46,7 \\
Solteiro & 76,7 \\
$>4$ anos estudados & 33,3 \\
Desempregado & 50,0 \\
\hline \hline
\end{tabular}

Resultados expresso por \%.

Os eventos sentinelas investigados fogem ao padrão etário observado em inquéritos de base populacional e de amplitude nacional realizados em capitais brasileiras. Possivelmente, esse padrão esteja relacionado ao tempo de uso de drogas, que variou de 1 a 54 anos e à média de 20,8 anos, também diferente da nacional, que é de 13 $\operatorname{anos}^{(19-20,25)}$.

O perfil etário da população em estudo não se assemelha ao de inquéritos transversais e indica que os eventos sentinelas e suas famílias, possivelmente, têm trajetória de recaídas e adoecimento, com usuários de drogas e famílias aproximando-se do "fundo do poço" e correlacionados à vulnerabilidade social. Esse perfil de uso prolongado, permeado de abstinências e recaídas, e de internações voluntárias e compulsórias, parece determinar a vida afetiva e conjugal, a escolaridade e a inserção no mercado de trabalho dos eventos sentinelas, pois a maioria não era casada, possuía baixa escolaridade e estava desempregada ou atuava em setores econômicos de baixa qualificação $0^{(10,20)}$.

A idade dos 30 familiares entrevistados variou de 19 a 78 anos, com média de 51,9 anos. A maioria era de mulheres $(86,7 \%)$, mães de usuários de álcool e outras drogas $(46,7 \%)$, e sete se encontravam em situação de chefe da família. Metade dos entrevistados era casado, portador de comorbidades crônicas, e a escolaridade predominante foi 0 Ensino Fundamental. Encontrou-se um número médio de 4,1 moradores por domicílio, com média de 1,8 criança e 1,4 idoso. Em relação às características do domicílio, a maior parte das famílias residia em casa própria, com média de seis cômodos, e cinco famílias $(16,7 \%)$ residiam em casa com até quatro cômodos, porém não houve distribuição espacial uniforme dos casos investigados nas regiões do município (Tabela 2). 
Tabela 2-Variáveis sociodemográficas e econômicas associadas às famílias sentinelas. Maringá (PR), Brasil, abril a setembro, 2014.

\begin{tabular}{lc}
\hline \hline Variáveis & Resultados \\
\hline Famílias & 30 \\
Crianças e adolescentes (0a 17 anos) & 20 \\
Idoso & 23 \\
Renda per capita & $689 \pm 652,06$ \\
Casa própria & 80,0 \\
Moradia em alvenaria & 70,0 \\
Moradia de madeira & 30,0 \\
Acesso a serviços de saúde exclusivamente do SUS & 33,3 \\
\hline \hline
\end{tabular}

Resultados expressos por $n$, média \pm desvio padrão ou $\%$.

SUS: Sistema Único de Saúde.

Das 30 famílias entrevistadas, $66,7 \%$ relataram que não tiveram dificuldade no atendimento, no serviço de saúde, no dia do evento, cinco famílias referiram que a maior dificuldade foi a demora para o atendimento, e uma informou descaso por parte dos profissionais, porque seu familiar encontrava-se sob efeito de drogas de abuso. Após a alta médica hospitalar, metade dos casos foi encaminhada para outros serviços de saúde ou para continuidade do tratamento ambulatorial. Apenas um usuário foi encaminhado para serviço de assistência psicossocial, para tratamento e reabilitação social.

$\mathrm{Na}$ avaliação do serviço hospitalar e da qualidade da assistência, $76,7 \%$ das famílias informaram que o serviço de saúde e a assistência prestada ao seu familiar foram ótimos e/ou bons; em quatro casos $(13,3 \%)$, foi regular, estando relacionado à queixa da demora de atendimento, à falta de informações sobre a evolução do quadro clínico do paciente e à jejum prolongado; e, em um caso $(3,3 \%)$, o familiar informou que o atendimento foi péssimo, pois ocorreram falhas de comunicação sobre aassistência.

Dos casos, $60 \%$ relataram que já procuraram ajuda para tratamento do uso de drogas de seu familiar. Apenas cinco $(27,7 \%)$ afirmaram que procuram o Centro de Atenção Psicossocial para Álcool e Drogas (CAPSad). A busca por tratamento e apoio profissional aconteceu em períodos que variaram, imediatamente à identificação do uso de drogas até 32 anos após, com média de 11,5 anos. A demora foi relatada por um familiar em decorrência da não aceitação do tratamento pelo usuário, em razão da vergonha e à dificuldade de aceitação do problema. Uma família referiu que acreditavam que "o uso fosse uma fase na vida [da pessoa] e que não seria prejudicial".

A utilização do CAPSad, considerado um dispositivo inovador para as estratégias de desinstitucionalização e humanização da Rede de
Atenção Psicossocial, foi referida por poucas famílias, durante a ocorrência de trauma por efeitos associados ao uso de álcool e outras drogas, atendidos em hospital geral; nenhum dos usuários estava em tratamento no CAPSad, quando aconteceu o trauma -diferente da taxa nacional, segundo a qual $50 \%$ das famílias conhecem os CAPSad e procuraram o atendimento para seus familiares nesses serviços de saúde ${ }^{(25)}$. Pode-se inferir, então, que as famílias dos eventos sentinelas, embora com necessidades de apoio profissional para assistência ao familiar doente, desconheciam os dispositivos disponíveis no município, reforçando a necessidade de implementação de uma rede articulada para inserção delas nos serviços de atenção a usuários de drogas.

Por meio da análise da causa raiz do uso de drogas pela operacionalização de um evento sentinela (uma metodologia de vigilância e investigação epidemiológica menos pragmática e mais qualitativa), ponderou-se que políticas públicas locorregionais, como acolhimento em serviços de Atenção Primária e CAPSad, eram deficientes na abordagem dos pacientes e de seus familiares.

O tratamento anterior ao evento traumático em $56,7 \%$ dos casos foi a internação hospitalar, em 52,9\% em hospital psiquiátrico. Cinco famílias (29,5\%) informaram que o tratamento foi pago diretamente à instituição de tratamento; duas relataram que essa situação afetou as finanças da família, que realizou empréstimo bancário e obteve ajuda da igreja. $\mathrm{O}$ número de internações variou de um a 20 , com média de 3,3 internações por usuário. Dos casos com história de internação hospitalar, a última teve duração de 2a 24 meses, com média de 6,8 meses.

Uma família informou que procurou tratamento ambulatorial em serviço de psicologia, porém, o familiar frequentou apenas seis seções e 
abandonou o tratamento. Os serviços de ajuda mútua foram informados por nove famílias (30\%), principalmente, serviços religiosos, e grupos Alcoólicos Anônimos e Amor Exigente. As demais famílias não referiram utilização de serviços de apoio e reabilitação.

As semelhanças e as diferenças dos eventos sentinelas estavam relacionadas ao contexto de vida do usuário e sua família, pois houve relatos de violência na infância, ocorrência de traumas anteriores, manobras ilícitas para aquisição da droga, alterações de comportamento no âmbito domiciliar e comportamento aditivo nas famílias (42\%).Em $20 \%$ das famílias, o uso de drogas acontecia dentro de casa. A razão para início do uso de drogas relatado pelo familiar em 18 casos (60\%) foi a influência de amigos e, em 17 deles $(56,8 \%)$, a família descobriu o uso imediatamente pela mudança de comportamento do usuário no contexto familiar.

A aproximação das causas do evento foi sintetizada em três momentos: primeira, aproximação ou fatores de risco para a iniciação do uso de drogas de abuso; segunda, aproximação ou fatores contributivos para a continuidade do uso de drogas de abuso; e terceira, aproximação ou determinação de falhas na Rede de Atenção de Saúde e Proteção Social.

No presente estudo, foi possível observar que a visão das famílias sobre a iniciação do uso de drogas pelos usuários pode ser, muitas vezes, atribuída a um fator singular ou até passar despercebida. A exposição a determinados fatores combinados, que poderiam contribuir para o uso de drogas de abuso, não é reconhecida pelas famílias. As famílias dos usuários investigados indicaram vários fatores de risco, isoladamente, para o início do uso de drogas de abuso, relacionados ao contexto familiar, aos grupos de pares (amigos), à escola, à discriminação social e à falta de serviços específicos de saúde.

Novamente, na ótica da maioria das famílias, não existiam justificativas objetivas. As famílias estudadas não possuíam estrutura para o enfrentamento do problema e nem tinham conhecimento suficiente sobre drogas e suas implicações, para lidar satisfatoriamente com o problema e compreender a necessidade de sua participação no processo terapêutico.

Outra situação observada foi a falta de assistência e comunicação social adequadas para o suprimento de uma necessidade considerada especial. As famílias desconhecem a rede assistencial do Sistema Único de Saúde (SUS) nessa área. Há carência de acesso a esses serviços, nos quais os usuários de drogas de abuso e suas famílias receberiam alternativas de tratamento e reinserção social, como a possibilidade de frequentar ambulatórios, comunidades terapêuticas e hospital-dia.

As más companhias, bastante citadas pelas famílias, evoluíram para o envolvimento com o tráfico de drogas, gerando a "dívida de droga" do usuário e lançando a alternativa do crime para pagá-la. A maioria das famílias investigadas, embora com renda familiar limitada, gozavam de situação financeira relativamente vantajosa, posto que adquiriram casa própria, ingressaram seus filhos na escola e tinham alimentação básica regular. No entanto, a droga de abuso e o ciclo de ação estabelecido pelos parâmetros envolvidos na tríade tráfico-violência-prisão eram determinantes sociais relevantes para a situação de vulnerabilidade em que essas famílias se encontravam.

Para a síntese dos antecedentes e dos fatores de risco, foi utilizado o item de conclusão do roteiro de investigação dos eventos sentinelas, que aponta evidências ou indícios de desvios das normas de prevenção no domicílio ou contexto familiar, no trabalho, na escola e nos serviços de saúde, terminando com a determinação de falhas e de oportunidades perdidas de acesso e acolhimento dos usuários e suas famílias, para que o processo fosse interrompido, ou seja, houvesse a interrupção da continuidade do uso de drogas de abuso que levou os jovens ao "fundo do poço".

Diante dessa caracterização, elaborou-se um modelo de síntese das causas subjacentes, categorizadas em contexto familiar, cultura/estilo de vida, educação, religião, atenção à saúde, assistência social, economia, e segurança pública com os respectivos fatores causais. (Figura 2) 
Figura 2- Categorias de fatores causais dos eventos sentinelas. Maringá (PR), Brasil, abril a setembro, 2014.

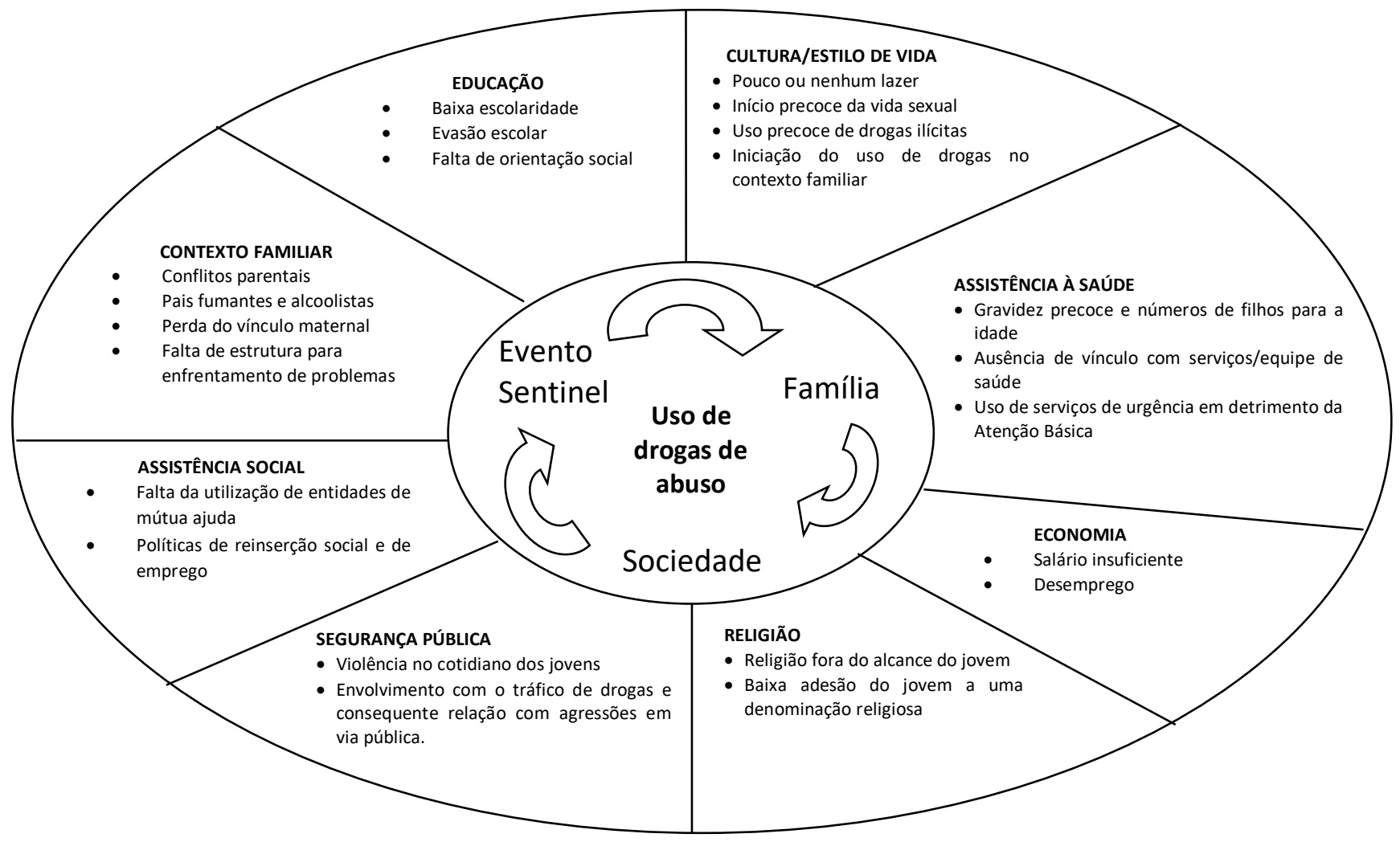

Fonte: Santana et al.(9)

A utilização do evento sentinela, por tratarse de um método de investigação epidemiológica que foge aos padrões tradicionais, permitiu obter muitas informações, a partir de um número reduzido de casos, possibilitando contribuir para o reconhecimento de fatores de risco em várias áreas e também para a definição de prioridades em ações preventivas do uso de drogas de abuso, envolvendo respostas de diversas políticas públicas.

Em todos os casos, encontraram-se, pelo menos, três aspectos estruturantes da vida dos usuários: uso associado de várias drogas de abuso, por longo período; evasão escolar e desemprego; e "fugas" constantes do lar, com pelo menos um episódio de situação de rua. Também foi recorrente o envolvimento em delitos e atos violentos mais de duas vezes.

Foram constatados elementos comuns e divergentes quanto à relação entre indivíduo/droga de abuso e suas consequências: tipo de família, condições socioeconômicas, modalidade de assistência à saúde, relações familiares e sociais, convivência com drogas de abuso na família e influência das drogas no cotidiano familiar. Em todas as famílias, foi possível observar antecedentes de risco e de proteção para o uso de drogas de abuso, em todos os domínios da vida e em qualquer nível de convivência socioambiental, o que parece indicar que a visão do problema restrita ao contexto familiar ou às características individuais dos usuários não seja suficiente.

Embora as condições de moradia da maioria das famílias fossem satisfatórias, a maior parte dos domicílios localizava-se em comunidades vulneráveis, confirmando que o consumo de drogas não ocorre de forma uniforme, socialmente ${ }^{(5,10)}$. As condições socioeconômicas das famílias podem determinar a trajetória assistencial na Rede de Atenção à Saúde e identificar o acesso e a utilização dos serviços $^{(10,21)}$.Aproximadamente, um terço das famílias dos eventos sentinelas utilizara a rede pública e as instituições filantrópicas de apoio social, para tratamento do uso de drogas, superando o índice de $14 \%$ constatado em estudos de base nacional ${ }^{(25)}$.

Para análise do evento sentinela e estabelecimento da causa raiz, foram estabelecidas categorias de fatores causais para discussão de critérios de evitabilidade com fatores: da comunidade e do paciente (casos em que ocorreu recusa em procurar a assistência ou 
em seguirem as orientações dos profissionais de saúde, por questões culturais e religiosas, ou por falta de reconhecimento do problema); profissionais (ausência de capacitação ou capacitação inadequada; nesses casos, devem-se tomar como referência as diretrizes dos manuais técnicos); institucionais (problemas políticoadministrativos, como falta de captação precoce e busca ativa dos casos, carência de leitos para tratamento e inexistência de sistema de referência e contrarreferência formalizado); sociais (condições socioeconômicas desfavoráveis, como desemprego, baixa renda familiar e baixa escolaridade do usuário e família); e intersetoriais (falta de equipamentos sociais e legais) $)^{(2,5,10)}$.

Como medida indireta da qualidade, a análise da tendência de eventos sentinelas aproxima os serviços de saúde das inadequações mais localizadas, principalmente em relação aos fracassos $^{(13,18)}$. Os agravos escolhidos como eventos sentinelas podem ser evitados em três diferentes níveis: pela organização social, com o acesso adequado a bens e serviços essenciais para toda a população; pelas medidas voltadas a eliminar ou diminuir fatores de risco específicos; e pelo acesso e utilização adequada de assistência à saúde de boa qualidade.

A interface entre políticas de educação, segurança pública, assistência social, economia e saúde parece determinar a ocorrência do uso de drogas de abuso nos casos investigados. A ausência de suporte social para a melhora das condições de vida do usuário e de suas famílias suporte social, aqui entendido como emprego, estabilidade do núcleo familiar e disponibilidade de rede de tratamento adequado - e a deficiência no acesso e no vínculo aos serviços de saúde, pouco acessíveis àquelas pessoas que mais necessitam, agravam essa situação. Para minimizar esse quadro, a parceria prevençãovigilância-assistência é preconizada para todos os casos.

O conceito da ocorrência em estudo como evento sentinela obriga a investigação mais qualitativa e com profundidade de cada ocorrência, tida como evitável no nível individual ou coletivo, propondo-se medidas pertinentes. Sua aplicação ao uso nocivo de drogas de abuso não pode se restringir à atenção individual, uma vez que as intervenções se organizam em bases populacionais e são provocadas por fatores econômicos e políticos. Deve-se implicar que a rede de serviços amplie sua cobertura nas áreas de maior incidência e, se o condicionamento socioeconômico problemas de saúde é real, também é responsabilidade dos sistemas de saúde ser mais acessíveis, nos quais os riscos são mais elevados.

$\mathrm{O}$ reconhecimento dos fatores de risco e o conhecimento precoce de problemas advindos do uso de drogas de abuso reforçam a necessidade da cadeia de intervenção, podendo evitar seu agravamento. $\mathrm{O}$ acompanhamento multidisciplinar e o apoio familiar representam ações importantes na prevenção de recaídas.

Neste estudo, apresentam-se limitações, por investigar dados de um CIAT, que corresponde a uma realidade específica, no entanto, o método da RCA permitiu análise mais profunda, identificando diversos fatores contribuintes e recomendações para evitar sua recorrência, o que supera essa fragilidade. Para chegar a esse objetivo, as informações coletadas mediante documentos, registros de atendimentos ambulatoriais, internações hospitalares e visitas domiciliares foram, cuidadosamente, exploradas e analisadas com rigor ético e profundidade.

As pesquisas epidemiológicas contribuem para a construção do conhecimento dos profissionais de enfermagem relacionadas às necessidades do usuário e de famílias que convivem com ocorrências relacionadas às drogas de abuso, à violência e a traumas - agravos estes relacionados a problemas de ordem social, de saúde e segurança pública.

O estudo destaca, por fim, a importância do enfermeiro como promotor do cuidado, devendo atuar junto dos usuários, familiares e comunidade, identificando possíveis agravos associados ao abuso de drogas, conflitos familiares e vulnerabilidades, colocando em prática ações de planejamentos, e agindo com ações baseadas nas políticas públicas existentes com enfoque na prevenção e na redução de danos.

\section{CONSIDERAÇÕES FINAIS}

O presente evento sentinela alcançou casos graves de uso de drogas de abuso e permitiu inferir que a maioria dos pacientes acessa serviços de saúde somente quando apresenta complicações relacionadas ao consumo compulsivo, com comprometimento clínico, em decorrência do uso crônico ou a situações de violência e trauma. Foi possível identificar que a abordagem psicossocial precoce não ocorreu frente a esses casos, permitindo categorizá-los como evitáveis. 
A investigação dirigida para a trajetória da ocorrência do evento, a identificação dos pontos críticos do processo da atenção à saúde e à vulnerabilidade social, e a possibilidade do fornecimento de visibilidade do processo permitem a crítica sobre o desempenho das políticas públicas. A interface entre políticas de educação, segurança pública, assistência social, economia e saúde, inadequadas e insuficientes, parece determinar a causa raiz do uso de drogas de abuso nos casos investigados.

\section{REFERÊNCIAS}

1- Crowley R, Kirschener N, Dunn AS, Bornsteins SS. Health and public policy to facilitate effective prevention and treatment of substance use disorders involving illicit and prescription drugs: An American College of Physicians position paper. Ann Intern Med. 2017;166(10):733-6.DOI: 10.7326/M16-2953

2- Macinko J. Avanços na vigilância da saúde pública brasileira. Ciênc Saúde Coletiva 2015;20(3):6286-9. DOI: 10.1590/141381232015203.20092014

3- Reis LM, Oliveira ML. Drugs and violence: Social perception in a community. Rev Eletr Enferm. 2015;17(3):1-9. DOI: 10.5216/ree.v17i3.28663

4- Sakae TM, Fernandez EA, Magajewski FR. Análise do perfil das internações hospitalares por drogadição em Santa Catarina entre 19982015. Arq Catarin Med. 2018 [citado em 1 abr 2020]; 47(3):16-37. Available in: http://www.acm.org.br/acm/seer/index.php/arq uivos/article/viewFile/306/270

5- Siqueira SA, Hollanda E, Motta JI. Equity promotion policies in health for vulnerable groups: The role of the Ministry of Helth. Ciênc Saúde Coletiva 2017;22(5):1397-406. DOI: 10.1590/1413-81232017225.33552016

6- Howlett M, Goetz KH. Introduction: Time, temporality and timescapes in administration and policy. International Int Rev Adm Sci. 2014;80(3):477-92. DOI: 10.1177/0020852314543210

7- Sales CC, Silva LF, Reis LM, Decesaro MN, Marcon SS, Oliveira ML. Social risk for the family of drug users hospitalized due to physical injury. J Res FundamCare 2019;11(2):510-6. DOI: 10.9789/2175-5361.2019.v11i2.510-516

8- Luz M, Corina J. Brasil, México, Colômbia e o combate ao narcotráfico no período de 2005 a 2017. In: Anais da 3a Semana Acadêmica de
Relações Internacionais da, 2018; Foz do Iguaçu. Foz do Iguaçu: UNILA; 2018. p. 93-106.

9- Santana CJ, Silvino MC, Rosa NM, Almeida EG, Reis LM, Oliveira ML. [Potentiality of a sentinel event for epidemiological surveillance of drug abuse]. J Nurs UFPE 2014;8(12):4337-44. DOI: 10.5205/reuol.6825-58796-1-SM.0812201419

10- Reis LM, Oliveira ML. Social vulnerability in families living with long-term addictive behavior. Acta Paul Enferm. 2017;30(4):412-9. DOI: 10.1590/1982- 0194201700061

11- Brasil, Ministério da Saúde. Guia de vigilância em saúde. 3a ed. Brasília, DF: Ministério da Saúde; 2019.

12- Ruoff AB, Andrade SR, Piccoli T. O processo de análise da evitabilidade dos casos de óbito infantile e fetal: Estudo de caso único. Texto Contexto-Enferm. 2018;27(4): 1-8. DOI: 10.1590/0104-07072018004030017

13- Rutstein DD, Berenberg W, Chalmers TC, Child CG, Fishman AP, Perrin EB, et al. Measuring the quality of medical care: A clinical method. N Engl J Med. 1976;294(11):582-8. DOI: 10.1056/NEJM197603112941104

14- Rutstein DD, Mullan RJ, Frazier TM, Halperin WE, Melius JM, Sestito JP. Sentinel health events (occupational): A basis for physician recognition and public health surveillance. Arch Environ Health 1984;39(3):159-68. DOI: 10.1080/00039896.1984.9939518

15- Silva MS. Trajetória assistencial de um evento sentinela: Avaliação da atenção integral ao usuário diabético no SUS a partir da retinopatia diabética grave [dissertação]. Pernambuco: Centro de Pesquisas Aggeu Magalhães da Fundação Oswaldo Cruz; 2015.

16- Bochner R. [Occupational fatality by pesticide exposre as a sentinela event: When a little menos a lot]. Vigil Sanit Debate 2015:1-11. DOI: 10.3395/2317-269x.00364

17- Ballani TS, Oliveira ML. [Use of drugs of abuse and sentinel event: Constructing a proposition about assessing public policies]. Texto ContextoEnferm. 2007;16(3):488-94. DOI: 10.1590/S010407072007000300015

18- Sillvino MC, Rosa NM, Santos JA, Seleghim MR, Ballani TS, Oliveira ML. [Operation of sentinela event to surveillance of drug abuse]. Healt Social Change 2012 [citado em 15 jan 2019]; 3(2):59-66. Available in: http://stat.saudeetransformacao.incubadora.ufsc .br/index.php/saudeetransformacao/article/view /1145 
19- Santana CJ, Oliveira ML. Effects of drug involvement on long-term users' family members. Rev RENE 2017;18(5):671-8. DOI: 10.15253/2175-6783.2017000500015

20- Santana CJ, Reis LM, Almeida EG, Oliveira ML. Caracterización sociodemográfica y estándar de uso de drogas en internados por trauma físico. Rev Presencia 2018 [citado em 1 abr 2020]; 14. Available in: http://ciberindex.com/index.php/p/article/view/ e11811

21- Reis LM, Gavioli A, Antunes F, Silvino MC, Rosa NM, Oliveira ML. Profile of users intoxicated by drugs of abuse and association with death. Rev RENE 2016;17(2):260-7. DOI: 10.1590/19820194201400019

22- Teixeira TC, Cassiani SH. Análise de causa raiz de acidentes por quedas e erros de medicação em hospital. Acta Paul Enferm. 2014;27(2):100-7. DOI: 10.1590/1982-0194201400019

23- Hoffman C. Canadian root cause analysis framework: A tool for identifying and addressing the root causes of critical incidents in healthcare. Canadá: Canadian Patient Safety Institute; 2006.

24- Pena MM, Melleiro MM. The root cause analysis method for investigation of adverse events. J Nurs UFPE 2017;11(Suppl 12):5297-304. DOI: $\quad 10.5205 / 1981-8963-v 11 i 12 a 25092 p 5297-$ 5304-2017

25- Laranjeira R, Madruga CS, Pinsky I, Caetano R, Mitsuhiro SS, Castelo G (Orgs). II Levantamento Nacional de Álcool e Drogas (LENAD) - 2012. São Paulo: Instituto Nacional de Ciência e Tecnologia para Políticas Públicas de Álcool e Outras Droga; 2014.

Nota: Extraído da dissertação "Internação hospitalar e trauma: evento sentinela para monitoramento dos efeitos das drogas de abuso", Universidade Estadual de Maringá, 2015.

Recebido em: 25/10/19

Aprovado em: 20/04/20

Endereço de correspondência: Rua Luiz Lerco, 455 - Apto 1706 Torre Mozart, CEP 86.047-610 - Londrina - PR

Email: cleitonjsantana@hotmail.com 